\title{
On Functions of Integrals
}

By R. P. Gillespie, Glasgow University.

(Received and read 5th February, 1932.)

\section{\$1. Statement of the Problem.}

The object of this paper is to discuss conditions for a maximum or minimum of functions of integrals of the type

$$
\phi\left[\int_{x_{1}}^{x_{y}} f\left(x, y, y^{\prime}\right) d x, \int_{x_{1}}^{x_{2}} g\left(x, y, y^{\prime}\right) d x\right],
$$

employing the methods of the Calculus of Variations.

Let $D$ be a three-dimensional domain consisting of points $\left(x, y, y^{\prime}\right)$, such that $(x, y)$ lies in a given two-dimensional domain $d$, and $y^{\prime}$ is such that $-\infty<y^{\prime}<\infty$. Let $f\left(x, y, y^{\prime}\right), g\left(x, y, y^{\prime}\right)$ be two functions, which are continuous, and possess continuous partial derivatives of the first three orders with respect to the variables $x, y, y^{\prime}$, in the domain $D$. Let $P_{1}\left(x_{1}, y_{1}\right), P_{2}\left(x_{2}, y_{2}\right)$ be two fixed points of the domain $d$, and let the function $y=\psi(x)$ have the following properties:

(i) single-valued, continuous in the interval $\left(x_{1}, x_{2}\right)$;

(ii) continuous derivative $\psi^{\prime}(x)$ in $\left(x_{1}, x_{2}\right)$;

(iii) $y_{1}=\psi\left(x_{1}\right), y_{2}=\psi\left(x_{2}\right)$;

(iv) the point $(x, y)$, where $y=\psi(x), x_{1} \leqq x \leqq x_{2}$, lies entirely in the domain $d$.

The class of curves $y=\psi(x)$ is called the class of admissible curces, say the class $\{C\}$. We now consider the functionals

$$
\begin{aligned}
& F(\psi)=\int_{x_{1}}^{x_{2}} f\left\{x, \psi(x), \psi^{\prime}(x)\right\} d x, \\
& G(\psi)=\int_{x_{1}}^{x_{2}} g\left\{x, \psi(x), \psi^{\prime}(x)\right\} d x .
\end{aligned}
$$

Let $F_{1}$ and $F_{2}, G_{1}$ and $G_{2}$ be the upper and lower bounds of the functionals $F$ and $G$ respectively, in the class $\{C\}$. We now introduce the function $\phi(p, q)$, which is a given continuous function of the variables $p$ and $q$ for the range

$$
F_{2} \leqq p \leqq F_{1}, \quad G_{2} \leqq q \leqq G_{1},
$$

and possesses continuous partial derivatives, with respect to $p$ and $q$, of the first two orders, for the same range. 
The functional, which it is proposed to discuss, is thus

$$
I(\psi)=\phi[F(\psi), G(\psi)],
$$

and conditions are found for a maximum or minimum of $I(\psi)$ in the class of curves $\{C\}$.

\section{§2. The Analogue of Euler's Equation.}

The neighbourhood $(k)$ of the curve $y=\psi_{0}(x)$ is defined to be that part of the $(x, y)$ plane bounded by the curves

$$
y=\psi_{0}(x)-k, \quad y=\psi_{0}(x)+k,
$$

and the straight lines $x=x_{1}$, and $x=x_{2}$. If it is possible to find a number $k$, such that

$$
I(\psi) \geqq I\left(\psi_{0}\right)
$$

for all admissible curves $\psi$, which lie entirely in the neighbourhood (k) of the curve $y=\psi_{0}(x)$, then the curve $y=\psi_{0}(x)$ is said to define a relative minimum of the functional $I(\psi)$. Thus the condition for a relative minimum is that the total variation

$$
\Delta I=I(\psi)-I\left(\psi_{0}\right)
$$

shall be positive or zero.

Proceeding in the manner usual in the Calculus of Variations, we have

$$
\begin{array}{r}
\Delta I=\phi\left[\int_{x_{1}}^{x_{2}} f\left(x, y+\Delta y, y^{\prime}+\Delta y^{\prime}\right) d x, \int_{x_{1}}^{x_{2}} g\left(x, y+\Delta y, y^{\prime}+\Delta y^{\prime}\right) d x\right] \\
-\phi\left[\int_{c_{1}}^{x_{2}} f\left(x, y, y^{\prime}\right) d x, \int_{y_{1}}^{x_{2}} g\left(x, y, y^{\prime}\right) d x\right] \\
=\phi_{F}[F, G] \quad\left[\int_{x_{1}}^{x_{2}} f\left(x, y+\Delta y, y^{\prime}+\Delta y^{\prime}\right) d x-\int_{x_{1}}^{x_{2}} f\left(x, y, y^{\prime}\right) d x\right] \\
+\phi_{G}[F, G] \quad\left[\int_{x_{2}}^{x_{2}} g\left(x, y+\Delta y, y^{\prime}+\Delta y^{\prime}\right) d x-\int_{x_{1}}^{x_{2}} g\left(x, y, y^{\prime}\right) d x\right] \\
+\frac{1}{2} \phi_{F F}[\ldots]\left[\int_{x_{1}}^{x_{2}} f\left(x, y+\Delta y, y^{\prime}+\Delta y^{\prime}\right) d x-\int_{x_{1}}^{x_{2}} f\left(x, y, y^{\prime}\right) d x\right]^{2} \\
+\phi_{F G}[\ldots] \quad\left[\int_{x_{2}}^{x_{2}} f\left(x, y+\Delta y, y^{\prime}+\Delta y^{\prime}\right) d x-\int_{x_{1}}^{x_{2}} f\left(x, y, y^{\prime}\right) d x\right] \\
\times \quad\left[\int_{x_{1}}^{x_{2}} g\left(x, y+\Delta y, y^{\prime}+\Delta y^{\prime}\right) d x-\int_{x_{1}}^{x_{2}} g\left(x, y, y^{\prime}\right) d x\right] \\
+\frac{1}{2} \phi_{G G}[\ldots]\left[\int_{x_{1}}^{x_{2}} g\left(x, y+\Delta y, y^{\prime}+\Delta y^{\prime}\right) d x-\int_{c_{1}}^{x_{2}} g\left(x, y, y^{\prime}\right) d x\right]^{2},
\end{array}
$$


where the arguments in $\phi_{F F}, \phi_{F G}, \phi_{G G}$ are

$$
\int_{x_{1}}^{x_{2}} f\left(x, y+\theta \Delta y, y^{\prime}+\theta \Delta y^{\prime}\right) d x \text { and } \int_{x_{1}}^{x_{2}} g\left(x, y+\theta \Delta y, y^{\prime}+\theta \Delta y^{\prime}\right) d x
$$

where $0<\theta<1$.

Let $\Delta y=\epsilon \eta(x)$, where $\eta(x)$ is any continuous function with a continuous derivative in the interval $\left(x_{1}, x_{2}\right)$, such that

$$
\eta\left(x_{1}\right)=0, \quad \eta\left(x_{2}\right)=0, \quad|\epsilon| \leqq \epsilon_{1},
$$

where $\epsilon_{1}$ is a fixed positive number, chosen so that the maximum value of $|\epsilon \eta(x)|$ in the interval $\left(x_{1}, x_{2}\right)$ is less than $k$. With these conditions, it is clear that the curve

$$
y=\psi_{0}(x)+\epsilon \eta(x)
$$

is an admissible curve lying entirely in the neighbourhood $(k)$ of the curve $y=\psi_{0}(x)$. Then

$$
\begin{aligned}
\Delta I & =\phi_{F}[F, G]\left\{\epsilon \int_{x_{1}}^{x_{2}}\left(\eta f_{y}+\eta^{\prime} f_{y^{\prime}}\right) d x\right. \\
& \left.+\frac{1}{2} \epsilon^{2} \int_{\prime_{1}^{\prime}}^{\prime_{2}}\left(\eta^{2} \frac{\partial^{2}}{\partial y^{2}}+2 \eta \eta^{\prime} \frac{\partial^{2}}{\partial y \partial y^{\prime}}+\eta^{\prime 2} \frac{\partial^{2}}{\partial y^{\prime 2}}\right) f\left(x, y+\theta^{\prime} \epsilon \eta, y^{\prime}+\theta^{\prime} \epsilon \eta^{\prime}\right) d x\right\} \\
& +\phi_{G}[F, G]\left\{\epsilon \int_{x_{1}}^{x_{2}}\left(\eta g_{y^{\prime}}+\eta^{\prime} g_{y^{\prime}}\right) d x+\ldots\right\} \\
& +\frac{1}{2} \phi_{F F}[\ldots]\left\{\epsilon \int_{x_{1}}^{x_{2}}\left(\eta f_{y}+\eta^{\prime} f_{y^{\prime}}\right) d x+\ldots\right\}^{2} \\
& +\phi_{F G}[\ldots]\left\{\epsilon \int_{x_{2}}^{x_{2}}\left(\eta f_{y}+\eta^{\prime} f_{y^{\prime}}\right) d x+\ldots\right\} \\
& \times\left\{\epsilon \int_{x_{1}}^{x_{2}}\left(\eta g_{y}+\eta^{\prime} g_{y^{\prime}}\right) d x+\ldots\right\} \\
& +\frac{1}{2} \phi_{G G}[\ldots]\left\{\epsilon \int_{x_{1}}^{x_{2}^{7}}\left(\eta g_{y}+\eta^{\prime} g_{y^{\prime}}\right) d x+\ldots\right\}^{2} .
\end{aligned}
$$

Since the functions involved are all continuous, the coefficients of $\epsilon^{2}, \epsilon^{3}, \epsilon^{4}$ in $\Delta I$ are all bounded; thus, by choosing $\epsilon$ small enough, the sign of $\Delta I$ is the same as that of

$$
\epsilon\left\{\phi_{F}[F, G] \int_{z_{1}}^{x_{2}}\left(\eta f_{y}+\eta^{\prime} f_{y^{\prime}}\right) d x+\phi_{G}[F, G] \int_{x_{2}}^{x_{2}}\left(\eta g_{y}+\eta^{\prime} g_{y^{\prime}}\right) d x\right\} \text {. }
$$

Hence a necessary condition that $y=\psi_{0}(x)$ should give a relative maximum or minimum is that, for every function $\eta(x)$, such that $\eta(x)$ and $\eta^{\prime}(x)$ are continuous in $\left(x_{1}, x_{2}\right)$ and $\eta\left(x_{1}\right)=\eta\left(x_{2}\right)=0$,

$$
\phi_{F}[F, G] \int_{c_{1}}^{x_{2}}\left(\eta f_{y}+\eta^{\prime} f_{y^{\prime}}\right) d x+\phi_{G}[F, G] \int_{c_{1}}^{c_{2}}\left(\eta g_{y}+\eta^{\prime} g_{y^{\prime}}\right) d x=0
$$


where

$$
\begin{aligned}
& F=\int_{x_{1}} f\left\{x, \psi_{0}(x), \psi_{0}{ }^{\prime}(x)\right\} d x, \\
& G=\int_{1}^{\prime} g\left\{x, \psi_{0}(x), \mid \psi_{0}{ }^{\prime}(x)\right\} d x .
\end{aligned}
$$

By applying the method of integration by parts to the above equation, we deduce

$$
\phi_{F}[F, G] \int_{x_{1}}^{x_{*}} \eta\left(f_{v^{\prime}}-\frac{d}{d x} f_{y^{\prime}}\right) d x+\phi_{G}[F, G] \int_{x_{1}}^{x_{2}} \eta\left(g_{n}-\frac{d}{d x} g_{y^{\prime}}\right) d x=0,
$$

which, by the Fundamental Lemma of the Calculus of Variations, gives

$$
\phi_{F}[F, G]\left\{f_{y}-\frac{d}{d x} f_{z^{\prime}}\right\}+\phi_{i z}[F, G]\left\{g_{y^{\prime}}-\frac{d}{d x} g_{y^{\prime}}\right\}=0 .
$$

Thus a first necessary condition to be satisfied by $y=\psi_{0}(x)$, in order that it should give a maximum or minimum of $I(\psi)$, is that it should satisfy the integro-differential equation (a), which is the analogue of Euler's Equation.

\section{§3. The Solution of Equation (a).}

If $y=y(x)$, is any solution of equation $(\alpha)$, the expression

$$
\phi_{F}\left[\int_{x_{1}}^{x_{2}} f\left(x, y, y^{\prime}\right) d x, \int_{x_{1}}^{x_{2}} g\left(x, y, y^{\prime}\right) d x\right],
$$

where $y=y(x)$, has a definite value, $\lambda_{1}$ say, independent of $x$; similarly $\phi_{G}[]$ has the value $\lambda_{2}$. Thus $y=y(x)$ satisfies the differential equation

$$
\lambda_{1}\left(f_{y^{\prime}}-\frac{d}{d x} f_{!^{\prime \prime}}\right)+\lambda_{2}\left(g_{!^{\prime \prime}}-\frac{d}{d x} g_{\prime^{\prime}}\right)=0,
$$

and we see, that each solution of equation $(\alpha)$ is also a solution of a certain differential equation of the second order. Let the solution of equation (1) be

$$
y=\psi\left(x, \alpha, \beta, \lambda_{1}, \lambda_{2}\right),
$$

where $\alpha$ and $\beta$ are constants of integration, and substitute this value for $y$ in

$$
\phi_{F}[]=\lambda_{1} \text { and } \phi_{G}[]=\lambda_{\underline{m}} .
$$

This gives us two equations to solve for $\lambda_{1}$ and $\lambda_{2}$, and remembering $y_{1}=y\left(x_{1}\right), y_{2}=y\left(x_{2}\right)$, we can find $a$ and $\beta$, thus obtaining a solution 
satisfying all the conditions. This is a complete solution of Equation (a), for it has been shown that every solution can be obtained in this way. It is important to notice that equations (2) may give one or more sets of values of $\lambda_{1}$ and $\lambda_{2}$, and therefore, a corresponding number of curves joining $P_{1}$ and $P_{2}$; also it has been tacitly assumed that

$$
\lambda_{1} f_{y^{\prime} y^{\prime}}+\lambda_{2} g_{y^{\prime} y^{\prime}} \neq 0 \text { in }\left(x_{1}, x_{2}\right) \text {. }
$$

As an illustration of the method consider the following example.

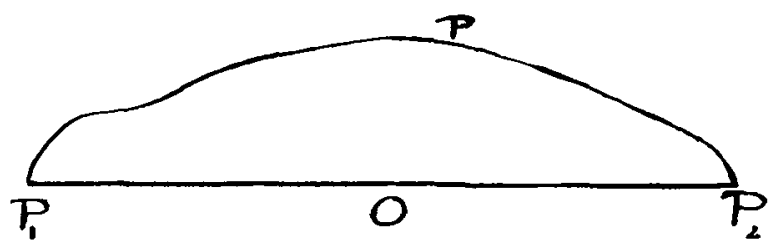

Amongst the admissible curves joining the points $P_{1}(-1,0)$ and $P_{2}(1,0)$, find one which gives a minimum to the functional

$$
I \equiv \frac{\left(\text { Length of } P_{1} P P_{2}\right)^{2}}{\text { Area } P_{1} O P_{2} P P_{1}}=\frac{\left[\int_{-1}^{1} \sqrt{ }\left(1+y^{\prime 2}\right) d x\right]^{2}}{\int_{-1}^{1} y d x} .
$$

We see that equation (a) for this problem is

$$
2 \frac{\int_{-1}^{1} \sqrt{ }\left(1+y^{\prime 2}\right) d x}{\int_{-1}^{1} y d x}\left[-\frac{d}{d x} \frac{y^{\prime}}{\sqrt{ }\left(1+y^{\prime 2}\right)}\right]-\frac{\left[\int_{-1}^{1} \sqrt{ }\left(1+y^{\prime 2}\right) d x\right]^{2}}{\left[\int_{-1}^{1} y d x\right]^{2}}=0 .
$$

Let

$$
\lambda=\frac{1}{2} \frac{\int_{-1}^{1} \sqrt{ }\left(1+y^{\prime 2}\right) d x}{\int_{-1}^{1} y d x}
$$

so that the differential equation is

$$
\frac{d}{d x} \frac{y^{\prime}}{\sqrt{\left(1+y^{\prime 2}\right)}}=-\lambda
$$

Thus the radius of curvature is numerically equal to $1 / \lambda$, and the solution is the arc of a circle. The next step is to find $\lambda$; if $P_{1} P P_{2}$ be the arc of a circle, radius $1 / \lambda$, then

$$
\lambda=\frac{1 / \lambda \sin ^{-1}(\lambda)}{1 / \lambda^{2} \sin ^{-1}(\lambda)-\sqrt{ }\left(1 / \lambda^{2}-1\right)} .
$$

On solving this equation for $\lambda$ we see that the radius of the circle is either infinity or 1. The first case is obviously not a minimum, and the required curve is therefore the semi-circle on $P_{1} P_{2}$ as diameter. 


\section{\$4. The Analogue of Legendre's Condition.}

If Equation $(\alpha)$ is satisfied, the coefficient of $\epsilon$ in the expansion of $\Delta I$ is zero, and it follows, as a necessary condition for a minimum of $I$, that the coefficient of $\epsilon^{2}$ in the expansion of $\Delta I$ must be positive or zero. Denoting this coefficient by $\frac{1}{2} I^{\prime \prime}$, we have, from $\S 2$;

$$
\begin{aligned}
& I^{\prime \prime}=\phi_{F}[F, G] \int_{x}^{x_{2}}\left(f_{m, \prime} \eta^{2}+2 f_{i \prime \prime}^{\prime} \eta \eta^{\prime}+f_{* \prime \prime \prime}^{\prime \prime} \eta^{2}\right) d x \\
& +\phi_{G}[F, G] \int_{x_{1}}^{x_{:}}\left(g_{\eta,} \eta^{2}+2 g_{\eta \eta^{\prime}} \eta \eta^{\prime}+g_{\eta^{\prime} \eta^{\prime}} \eta^{\prime 2}\right) d x \\
& +\phi_{F F}[\ldots]\left[\int_{x_{1}^{\prime}}^{x_{2}}\left(f_{\eta \prime} \eta+f_{\prime^{\prime}} \eta^{\prime}\right) d x\right]^{2} \\
& +2 \phi_{F G}[\ldots]\left[\int_{x_{1}}^{x_{*}}\left(f_{\prime \prime} \eta+f_{y^{\prime}} \eta^{\prime}\right) d x\right]\left[\int_{x_{1}}^{v_{2}}\left(g_{y} \eta+g_{y^{\prime}} \eta^{\prime}\right) d x\right] \\
& +\phi_{G G}[\ldots]\left[\int_{x_{1}}^{\ddots_{2}}\left(g_{y} \eta+g_{y^{\prime}} \eta^{\prime}\right) d x\right]^{2} \text {. }
\end{aligned}
$$

Let

$$
\begin{aligned}
& P \equiv \phi_{F}[F, G] f_{y y^{\prime}}+\phi_{G}[F, G] g_{y y^{\prime}}, \\
& Q \equiv \phi_{F}[F, G] f_{y y^{\prime}}+\phi_{G}[F, G] g_{y \prime^{\prime}}, \\
& R \equiv \phi_{F}[F, G] f_{y^{\prime} y^{\prime}}+\phi_{G}[F, G] g_{y^{\prime} y^{\prime}},
\end{aligned}
$$

and suppose that at some point $c$ in $\left(x_{1}, x_{2}\right), R$ is negative. Since all the functions involved are continuous, we can find a positive number $\delta$, such that $R(x)<0$ for values of $x$ in the interval

$$
\xi_{1}=c-\delta \leqq x \leqq c+\delta=\xi_{2} .
$$

Let $\eta(x)$ have the following special form

$$
\begin{aligned}
& \eta=0, x_{1} \leqq x \leqq \xi_{1} \\
& \eta=\left(x-\xi_{1}\right)^{2}\left(x-\xi_{2}\right)^{2}, \xi_{1} \leqq x \leqq \xi_{2} \\
& \eta=0, \xi_{2} \leqq x \leqq x_{2} .
\end{aligned}
$$

We now proceed to find the value of $I^{\prime \prime}$ for this special variation. The following results are used:

that

$$
\begin{aligned}
& \int_{\xi_{1}}^{\xi_{2}} P \eta^{2} d x=\frac{P\left(x^{\prime}\right)}{630}\left(\xi_{2}-\xi_{1}\right)^{9}, \xi_{1} \leqq x^{\prime} \leqq \xi_{2} \\
& \int_{\xi_{1}}^{\xi_{2}} 2 Q \eta \eta^{\prime} d x=2^{-7} \lambda\left(\xi_{2}-\xi_{1}\right)^{s}, \text { where } \lambda \text { is a constant such }
\end{aligned}
$$

$$
|\lambda| \leqq|Q(x)| \text { in } \xi_{1} \leqq x \leqq \xi_{2},
$$

(this result is obtained by dividing the range of $x$ into two equal parts); 


$$
\begin{aligned}
& \int_{\xi_{1}}^{\xi_{-}} R \eta^{\prime 2} d x=\frac{2 R\left(x^{\prime \prime}\right)}{105}\left(\xi_{2}-\xi_{1}\right)^{i}, \xi_{1} \leqq x^{\prime \prime} \leqq \xi_{2} ; \\
& \int_{\xi_{1}}^{\xi_{2}} f_{y} \eta d x=\frac{f_{y}\left(x^{\prime \prime \prime}\right)}{30}\left(\xi_{2}-\xi_{1}\right)^{5}, \xi_{1} \leqq x^{\prime \prime \prime} \leqq \xi_{2} ; \\
& \int_{\xi_{1}}^{\xi_{2}} g_{y^{\prime \prime}} \eta d x=\frac{g_{y}\left(x^{\mathrm{iv}}\right)}{30}\left(\xi_{2}-\xi_{1}\right)^{5}, \xi_{1} \leqq x^{\mathrm{iv}} \leqq \xi_{2} ; \\
& \int_{\xi_{1}}^{\xi_{2}} f_{y^{\prime}} \eta^{\prime} d x=2^{-3} \lambda_{1}\left(\xi_{2}-\xi_{1}\right)^{4},\left|\lambda_{1}\right| \leqq\left|f_{y^{\prime}}\right| \text { in } \xi_{1} \leqq x \leqq \xi_{2} ; \\
& \int_{\xi_{1}}^{\xi_{2}} g_{y^{\prime}} \eta^{\prime} d x=2^{-3} \lambda_{2}\left(\xi_{2}-\xi_{1}\right)^{4},\left|\lambda_{2} \leqq\right| g_{y^{\prime}} \mid \text { in } \xi_{1} \leqq x \leqq \xi_{2} .
\end{aligned}
$$

Thus we have

$$
\begin{aligned}
I^{\prime \prime} & =P\left(x^{\prime}\right) \frac{\left(\xi_{2}-\xi_{1}\right)^{\prime \prime}}{630}+2^{-7} \lambda\left(\xi_{2}-\xi_{1}\right)^{8}+R\left(x^{\prime \prime}\right) \frac{2}{105}\left(\xi_{3}-\xi_{1}\right)^{7} \\
& +\phi_{F F}[\ldots]\left[f_{y}\left(x^{\prime \prime \prime}\right) \frac{\left(\xi_{2}-\xi_{1}\right)^{5}}{30}+2^{-3} \lambda_{1}\left(\xi_{2}-\xi_{1}\right)^{4}\right]^{2} \\
& +2 \phi_{F G}[\ldots]\left[f_{y}\left(x^{\prime \prime \prime}\right) \frac{\left(\xi_{2}-\xi_{1}\right)^{5}}{30}+2^{-3} \lambda_{1}\left(\xi_{2}-\xi_{1}\right)^{4}\right] \\
& \times\left[g_{y \prime}\left(x^{\mathrm{iv}}\right) \frac{\left(\xi_{2}-\xi_{1}\right)^{5}}{30}+2^{-3} \lambda_{2}\left(\xi_{2}-\xi_{1}\right)^{4}\right] \\
& +\phi_{G G}[\ldots]\left[g_{l^{\prime}}\left(x^{\mathrm{iv}}\right) \frac{\left(\xi_{2}-\xi_{1}\right)^{5}}{30}+2^{-3} \lambda_{2}\left(\xi_{2}-\xi_{1}\right)^{4}\right]^{2}
\end{aligned}
$$

Since we can take $\delta$ as small as we please, and since all the functions involved are continuous, $I^{\prime \prime}$ is negative because $R\left(x^{\prime \prime}\right)$ is negative; hence a necessary condition that the curve $y=\psi_{0}(x)$ should give a minimum value to the functional $I$ is that $\phi_{F}[F, G] f_{y^{\prime} y^{\prime}}\left[x, \psi_{0}^{\prime}(x), \psi_{0}(x)\right]+\phi_{G}[F, G] g_{y^{\prime} y}\left[x, \psi_{0}(x), \psi_{0}^{\prime}(x)\right] \geqq 0, x_{1} \leqq x \leqq x_{2}$, where

$$
F \equiv \int_{x_{1}}^{x_{2}} f\left[x, \psi_{0}(x), \psi_{0}^{\prime}(x)\right] d x, \quad G \equiv \int_{x_{1}}^{x_{2}} g\left[x, \psi_{0}(x), \psi_{0}^{\prime}(x)\right] d x .
$$

This is the analogue of Legendre's Condition.

\section{§5. The Problem in the Parametric Notation.}

The previous results can be easily extended to the problem in the parametric notation. The functions $F\left(x, y, x^{\prime}, y^{\prime}\right), G\left(x, y, x^{\prime}, y^{\prime}\right)$ 
have the properties defined in Bolza's Vorlesungen über Variationsrechnung, pp. 193-197, and the problem is to find a curve joining the points $P_{1}$ and $P_{2}$, which will yield a minimum to the functional

$$
I=\phi\left[\int_{t_{1}}^{t_{2}} F\left\{x(t), y(t), x^{\prime}(t), y^{\prime}(t)\right\} d t, \int_{t_{1}}^{t_{2}} G\left\{x(t), y(t), x^{\prime}(t), y^{\prime}(t)\right\} d t\right]
$$

in the class of admissible curves

$$
x=x(t), y=y(t),
$$

joining the points $P_{1}$ and $P_{2}$.

We introduce the functions $F_{1}$ and $G_{1}$, defined in Bolza, p. 196, and by a reasoning similar to that of $\S 2$, we get the analogue of Euler's Equation:

$\phi_{F}[]\left(F_{x y^{\prime}}-F_{x^{\prime} y}\right)+\phi_{G}[]\left(G_{x y^{\prime}}-G_{x^{\prime} y}\right)+\left[\phi_{F}[] F_{1}+\phi_{G}[] G_{1}\right]\left(x^{\prime} y^{\prime \prime}-x^{\prime \prime} y^{\prime}\right)=0$.

This equation is solved similarly to that treated in $\S 3$. By a method analogous to that of $\S 4$, we obtain two further necessary conditions:

$$
\begin{aligned}
& \phi_{F}[] F_{x^{\prime} x^{\prime}}+\phi_{G}[] G_{x^{\prime} x^{\prime}} \geqq 0, \\
& \phi_{F}[] F_{y^{\prime} y^{\prime}}+\phi_{G}[] G_{y^{\prime} y^{\prime}} \geqq 0 ;
\end{aligned}
$$

both of these conditions are included in the one condition

$$
\phi_{F}[] F_{1}+\phi_{G}[\text {. }] G_{1} \geqq 0,
$$

which is the analogue of Legendre's Condition.

\section{§. Lower Semi-Continuity.}

[See Tonelli's Fondamenti di Calcolo delle Variazioni, Vol. I., Chaps. VI. and VII., where necessary and sufficient conditions for lower semi-continuity of the integral $\int_{C} F\left(x, y, x^{\prime}, y^{\prime}\right) d t$ are discussed.]

It is assumed here that such conditions are satisfied as to make the integrals

$$
\int F\left(x, y, x^{\prime}, y^{\prime}\right) d t, \int G\left(x, y, x^{\prime}, y^{\prime}\right) d t
$$

lower semi-continuous in a class of curves in a bounded part of the $(x, y)$ plane. [See Tonelli, Vol. I., pp. 201, 202, for definitions of the types of curves considered and the nature of the part of the plane.] Further, it is assumed that $\phi(p, q), \phi_{\rho}(p, q), \phi_{q}(p, q)$ are continuous, positive functions for a range of values of $p$ and $q$, which includes in the $p$ range all values of $\int_{C} F d t$ in the set of curves, and in the $q$ range all values of $\int_{C} G d t$ in the set of curves. 
With these conditions it will now be shown that

$$
I_{C} \equiv \phi\left[\int_{C} F d t, \int_{C} G d t\right]
$$

is lower semi-continuous.

Given a positive number $\epsilon$ there exist neighbourhoods $\left(\rho_{1}\right)$ and $\left(\rho_{2}\right)$ of the curve $C_{0}$, a member of the set, such that for every curve $C$ belonging to them "ordinately" [see Tonelli, Vol. I., p.72],

$$
\begin{aligned}
& \int_{e} F d t>\int_{C_{0}} F d t-\epsilon, \text { in }\left(\rho_{1}\right) ; \\
& \int_{C} G d t>\int_{C_{o}} G d t-\epsilon, \text { in }\left(\rho_{2}\right) .
\end{aligned}
$$

Thus there exists a neighbourhood of $C_{0}$, common to $\left(\rho_{1}\right)$ and $\left(\rho_{2}\right)$, in which both these inequalities hold. There are four possible cases to consider.

$$
\int_{t} F d t>\int_{C_{0}} F d t, \quad \int_{C} G d t>\int_{C_{o}} G d t
$$

in this case, since $\phi_{p}(p, q)$ and $\phi_{q}(p, q)$ are both positive,

$$
\begin{gathered}
\phi\left[\int_{C} F d t, \int_{C} G d t\right]>\phi\left[\int_{C_{n}} F d t, \int_{C_{0}} G d t\right] . \\
\int_{C_{0}} F d t \geqq \int_{C^{\prime}} F d t>\int_{C_{0}} F d t-\epsilon, \\
\int_{C_{0}} G d t \geqq \int_{C} G d t>\int_{C_{0}} G d t-\epsilon
\end{gathered}
$$

here

$$
\begin{array}{r}
\phi\left[\int_{C} F d t, \int_{C} G d t\right]>\phi\left[\int_{C_{n}} F d t-\epsilon, \int_{C_{o}} G d t-\epsilon\right] \\
=\phi\left[\int_{C_{\mathrm{o}}} F d t, \int_{C_{\mathrm{n}}} G d t\right]-\epsilon\left\{\phi_{F}[]+\phi_{G}[]\right\},
\end{array}
$$

where $\phi_{F}$ and $\phi_{G}$ have arguments

$$
\left[\int_{C_{0}} F d t-\theta \epsilon, \int_{C_{\mathrm{u}}} G d t-\theta \epsilon\right], \quad 0<\theta<1 .
$$

Let $\delta$ be the minimum value of $\left[\phi_{F}+\phi_{G}\right]$ in the given range of values where $\delta>0$; then

$$
\phi\left[\int_{C} F d t, \int_{C} G d t\right]>\phi\left[\int_{c_{0}} F d t, \int_{C_{0}} G d t\right]-\epsilon \delta .
$$

(iii) $\int_{C} F d t>\int_{C_{v}} F d t$

$$
\int_{C_{0}} G d t \geqq \int_{C} G d t>\int_{C_{0}} G d t-\epsilon ;
$$


then

But

$$
\begin{aligned}
\phi\left[\int_{C} F d t, \int_{C} G d t\right]-\phi\left[\int_{C_{0}} F d t, \int_{C_{o}} G d t\right] \\
=\phi\left[\int_{C} F d t, \int_{C} G d t\right]-\phi\left[\int_{C_{0}} F d t, \int_{C} G d t\right] \\
+\phi\left[\int_{C_{0}} F d t, \int_{C} G d t\right]-\phi\left[\int_{C_{0}} F d t, \int_{C_{0}} G d t\right] .
\end{aligned}
$$

and

$$
\phi\left[\int_{C} F d t, \int_{C} G d t\right]>\phi\left[\int_{C_{0}} F d t, \int_{C} G d t\right]
$$

$$
\phi\left[\int_{C_{0}} F d t, \int_{C} G d t\right]>\phi\left[\int_{C_{0}} F d t, \int_{C_{n}} G d t\right]-\epsilon \delta^{\prime}
$$

where $\delta^{\prime}$ is a positive number, by (ii).

$$
\int_{C_{\mathrm{o}}} F d t \geqq \int_{c} F d t>\int_{C_{0}} F d t-\epsilon, \quad \int_{C} G d t>\int_{C_{0}} G d t
$$

which is exactly similar to (iii).

All possible cases have been considered, and it has been shown that, with the above conditions,

$$
\phi\left[\int_{C} F d t, \int_{C} G d t\right]
$$

is a lower semi-continuous function.

The above method can be applied to the following case. Suppose that $\int_{C} F d t$ is lower semi-continuous, and $\int_{C} G d t$ upper semicontinuous, while $\phi_{p}(p, q)$ is positive and $\phi_{q}(p, q)$ is negative for the given range of values; then, with these conditions, by considering the four possible cases by the previous method, it can be easily shown that

is lower semi-continuous.

$$
\phi\left[\int_{c} F d t, \int_{c} G d t\right]
$$

\section{§7. Existence of an Absolute Minimum.}

Let $F\left(x, y, x^{\prime}, y^{\prime}\right), G\left(x, y, x^{\prime}, y^{\prime}\right)$ be two functions, which are positive for every point of a bounded part of the $(x, y)$ plane, and for every $\left(x^{\prime}, y^{\prime}\right)$ such that $x^{\prime}$ and $y^{\prime}$ are not both zero. It is further assumed that the derived functions $F_{1}$ and $G_{1}$, of $\S 5$, are not negative in the same domain. 
With these conditions, it will now be shown that in each complete class of ordinary curves $C$ in the given bounded part of the plane, there always exists an absolute minimum of the functional

$$
I_{C} \equiv \phi\left[\int_{c} F d t, \int_{C} G d t\right]
$$

where $\phi(p, q), \phi_{p}(p, q), \phi_{q}(p, q)$ are continuous, positive functions for a range of values, which include, as above, all possible values of $\int_{c} F d t$ and $\int_{c} G d t$. By the results of Tonelli, Vol, I., Chap. VII., the functionals $\int_{C} F d t, \int_{C} G d t$ are lower semi-continuous, and hence, by the previous paragraph, it is seen that $I_{C}$ is lower semi-continuous.

There exist positive numbers $m_{F}, M_{F}, m_{G}, M_{G}$, such that in the given domain

$$
m_{F} \leqq F \leqq M_{F}, \quad m_{G} \leqq G \leqq M_{G \cdot}
$$

There is no loss of generality in assuming $x^{\prime 2}+y^{\prime 2}=1$, and since $\phi_{p}$ and $\phi_{r}$ are both positive, it is clear that

$$
\phi\left[m_{F} L, m_{G} L\right] \leqq I_{c} \leqq \phi\left[M_{F} L, M_{G} L\right],
$$

where $L$ denotes the length of the curve $C$. Now let

$$
C_{1}, C_{\varepsilon}, \ldots, C_{n}, \ldots
$$

be a minimising sequence for $I_{C}$; then either

$$
I_{c_{n}} \leqq-n, \text { or } I_{c_{n}} \leqq i+\frac{1}{n},
$$

where $i$ is a positive number. But obviously $I_{C_{n}} \leqq-n$ cannot be true; hence

$$
I_{C_{n}} \leqq i+\frac{1}{n}
$$

Now let $u=\phi\left[m_{F} L, m_{G} L\right]$; then since $\phi_{p}$ and $\phi_{q}$ are both positive, we can solve this equation for $L$ in the form $\psi(u)$, where $\frac{d \psi(u)}{d u}$ is positive. But

therefore

$$
\phi\left[m_{F} L, m_{G} L\right] \leqq I_{c}
$$

$$
L \leqq \psi\left(I_{C}\right)
$$

If $L_{n}$ denotes the length of $C_{n}$, we have thus shown that

$$
L_{n} \leqq \psi\left(I_{C_{n}}\right) \leqq \psi(i+1)
$$


The curves of the set $\left\{C_{n}\right\}$ are thus of bounded length, since $\psi(i+1)$ is a fixed positive number, and we can deduce that the set $\left\{C_{n}\right\}$ admits of at least one curve of accumulation, $C_{0}$ say, which is rectifiable and continuous, and therefore, belongs to the set $\{C\}$.

Now $I_{C}$ is lower semi-continuous, and thus $I_{C_{b}}<I_{C}+\epsilon$. Hence $I_{C_{0}} \leqq i+\epsilon$, and, since $\epsilon$ is arbitrary, $I_{C_{0}} \leqq i$. But we have proved that $C_{0}$ belongs to the class $\{C\}$, which shows that $I_{C_{0}} \geqq i$. Therefore $I_{C_{\mathrm{o}}}=i$, thus proving that $I_{C_{0}}$ is an absolute minimum of $I_{C}$ in the set $\{C\}$, and that $C_{0}$ is a minimising curve.

The following case can also be treated by the same method:

$$
\begin{gathered}
F\left(x, y, x^{\prime}, y^{\prime}\right)>0, \quad G\left(x, y, x^{\prime}, y^{\prime}\right)<0 \\
F_{1}\left(x, y, x^{\prime}, y^{\prime}\right) \geqq 0, \quad G_{1}\left(x, y, x^{\prime}, y^{\prime}\right) \leqq 0 \\
\phi>0, \quad \phi_{p}>0, \quad \phi_{1}<0,
\end{gathered}
$$

With these conditions it can be shown that in each complete class of ordinary curves $C$ in the given bounded part of the plane, there always exists an absolute minimum of $I_{c}$.

There exist two positive numbers $m_{F}$ and $m_{G}$, such that, in the given domain

hence

$$
m_{F} \leqq F, \quad-m_{G} \geqq G ;
$$

$$
\phi\left[m_{F} L,-m_{G} L\right] \leqq I_{C} .
$$

If as before $\left\{C_{n}\right\}$ is a minimising sequence, it is clear that

$i$ being a positive number.

$$
I_{c_{n}} \leqq i+\frac{1}{n}
$$

Let $u=\phi\left[m_{F} L,-m_{G} L\right]$; then we have

$$
\frac{d u}{d L}=\phi_{p} m_{F}-m_{G} \phi_{q}>0 \text {, }
$$

and hence $\frac{d L}{d u}$ is always positive.

Thus we can solve for $L$ in the form $\psi(u)$, where $\psi^{\prime}(u)$ is positive; thus

$$
L \leqq \psi\left(I_{C}\right)
$$

and the rest of the proof follows exactly as before. 